\title{
1 Weekday and weekend sedentary time and physical activity in differentially active \\ 2 children
}

4 Objectives. To investigate whether weekday-weekend differences in sedentary time (ST) and

5 specific intensities of physical activity exist among children categorised by physical activity 6 levels.

7 Design. Cross-sectional observational study.

8 Methods. Seven-day accelerometer data were obtained from 810 English children $(n=420$

9 girls) aged 10-11 yr. Daily average $\min \bullet \mathrm{d}^{-1}$ spent in moderate to vigorous physical activity 10 (MVPA) were calculated for each child. Sex-specific MVPA quartile cut-off values 11 categorised boys and girls separately into four graded groups representing the least (Q1) 12 through to the most active (Q4) children. Sex- and activity quartile-specific multilevel linear 13 regression analyses analyzed differences in ST, light physical activity (LPA), moderate 14 physical activity (MPA), vigorous physical activity (VPA), and MVPA between weekdays 15 and weekends.

16 Results. On weekdays Q2 boys spent longer in LPA $(p<0.05)$, Q1 ( $p<0.001)$, Q2 boys

$17(p<0.01)$ did significantly more MPA, and Q1-Q3 boys accumulated significantly more VPA 18 and MVPA than at weekends. There were no significant differences in weekday and weekend

19 ST or physical activity for Q4 boys. On weekdays Q2 and Q3 girls accumulated more ST

$20(p<0.05), \mathrm{Q} 1$ and Q2 girls did significantly more MPA $(p<0.05)$, and Q1-Q3 girls engaged in 21 more VPA $(p<0.05)$ and more MVPA $(p<0.01)$ than at weekends. Q4 girls' ST and physical 22 activity varied little between weekdays and weekends. 
23 Conclusions. The most active children maintained their ST and physical activity levels at

24 weekends, while among less active peers weekend ST and physical activity at all intensities

25 was lower. Low active children may benefit most from weekend intervention strategies.

\section{$26 \underline{\text { Key words }}$}

27 High active, time-of-week differences, motor activity, health, child, multilevel analysis 
Introduction.

29 Regular physical activity (PA) during childhood provides an array of health benefits ${ }^{1}$.

30 Insufficient childhood PA and excessive sedentary time (ST) however are independently

31 associated with negative health outcomes such as obesity and cardiometabolic risk ${ }^{2}$. To establish healthy lifestyles in children efforts to increase PA and reduce ST are public health priorities. To maintain good health, guidelines state that school-age youth accumulate at least $60 \mathrm{~min} \bullet \mathrm{d}^{-1}$ of moderate-to-vigorous PA (MVPA) and reduce ST ${ }^{1}$.

Children's PA behaviours vary in bout frequency, duration, and intensity depending on the setting where they occur. For example, there is greater uniformity in school day activity compared to after-school and weekends, which allow more behavioural choice ${ }^{3}$. Within such contexts ST and PA levels can vary markedly due to the influence of multidimensional correlates $^{4-5}$. Perhaps unsurprisingly, children tend to be less sedentary and active at weekends than on weekdays ${ }^{6-8}$. Weekends present more discretionary time for ST and PA, but also lack the regular routines and structures of school weekdays which determine a significant proportion of children's daily ST and PA ${ }^{3}$. It is unclear though whether engagement of high and low active children in ST and PA differs between weekdays and weekends. Previous research found weekend PA to be lower than on weekdays regardless of children's PA classification ${ }^{9-11}$, but seldom have such trends been examined by intensityspecific PA ${ }^{11}$ or with ST as the outcome. Furthermore, weekends are an important context for 47 activity promotion but it is unknown whether targeting specific groups of children may be a more efficient and efficacious intervention approach than a population-based one. Thus, the study objectives were to investigate whether hypothesised differences in weekday and weekend ST and specific intensities of PA exist among English children categorised by their

51 PA levels. 
Methods.

53 Seventeen schools situated in a north-west England borough of over 300,000 people participated in this cross-sectional research during 2008, 2009, and 2010. Eight schools were recruited in 2008 and 2009 as part of a two year study of children's PA and health outcomes. In 2010 three of these schools were joined by nine others for the baseline phase of a schoolbased PA and nutrition intervention. In each year the same data collection procedures were applied and so for the purposes of this paper the data were aggregated. To be eligible to participate, children had to be in school Year 6 (aged 10-11 years) and be free from any physical disabilities preventing them for taking part in routine physical activities. A fixed available sample of all Year 6 children $(\mathrm{N}=992 ; 307$ in 2008, 295 in 2009, 390 in 2010) were informed of the research by their class teachers, received project and consent information, and were invited to participate. Written informed parental consent and child assent were received from 818 children $(230$ in 2008,270 in 2009, 318 in 2010; $82.5 \%$ participation rate). Children participated at one time point only (i.e., 2008, 2008, or 2010). The ethnic origin of the children was white British, which reflects the ethnic demographic of the borough's school-age population ${ }^{12}$. In each year data were collected in one school per week between October and December. Ethical approval was obtained from the University Ethics Committee for each year of study (reference numbers 8.56 and 10/ECL/039). Ethical principles of the Declaration of Helsinki were adhered to throughout this research.

Stature was measured to the nearest $0.1 \mathrm{~cm}$ using a portable stadiometer (Leicester Height Measure, Seca, Birmingham, UK). Body mass was measured to the nearest $0.1 \mathrm{~kg}$ using calibrated scales (Seca, Birmingham, UK) with the children in light clothing and barefooted. Body mass index (BMI) was calculated (body mass $(\mathrm{kg}) /$ stature $\left.^{2}\left(\mathrm{~m}^{2}\right)\right)$ and BMI z-scores 
cut-points were used to classify children as normal-weight or overweight/obese ${ }^{14}$. Home postal codes were used to generate indices of multiple deprivation (IMD) scores which indicated area-level socio-economic status (SES). IMD scores are a composite of seven domains of deprivation with higher scores representing higher degrees of deprivation ${ }^{15}$.

PA was objectively measured using ActiGraph accelerometers (GT1M and GT3x, ActiGraph LLC, Pensacola, FL) which were worn over the right hip for 7 consecutive days from waking until bedtime. Accelerometers were set to record data using 5 second epochs. ActiGraph cut points of 100 counts $\cdot \mathrm{min}^{-1}, 101-2295$ counts $\cdot \mathrm{min}^{-1}, 2296$ counts $\cdot \mathrm{min}^{-1}$, and 4012 counts $\cdot \mathrm{min}^{-1}$ classified the boundaries of ST, light intensity PA (LPA), moderate intensity PA (MPA) and vigorous intensity PA (VPA), respectively ${ }^{16}$. In the absence of universally agreed cut-points to classify children's PA intensities, the cut-points of Evenson et al. ${ }^{16}$ were selected on the basis of a methodologically rigorous comparison study, which concluded that they have acceptable classification accuracy across a range of intensities and are appropriate for use with 5-15 year olds ${ }^{17}$. MPA and VPA are influenced by different factors and both may affect health outcomes differently ${ }^{7}$. Taking these reasons together provided a rationale for studying MPA and VPA separately from overall moderate to vigorous PA (MVPA). Nonaccelerometer wear time was defined as at least 20 min periods of consecutive zero counts ${ }^{18}$. Wear time criteria were at least $540 \mathrm{~min} \bullet \mathrm{d}^{-1}$ on week days and $480 \mathrm{~min} \bullet \mathrm{d}^{-1}$ on weekend days, 96 for at least two week days and one weekend day. These criteria have been shown to yield a 97 reliability of 0.9 suggesting a high degree of consistency across days ${ }^{19}$. One hundred and seventy seven children did not achieve the wear time criteria, and technical failures downloading accelerometer data were experienced for a further 10 children. Missing data analysis was completed using missing at random (MAR) assumptions. There is no way to

101 directly test these assumptions but relationships between health-related variables and missing 
102 data can indicate whether they hold true ${ }^{20}$. In our sample there were no differences in BMI,

103 BMI z-score, weight status, and SES between the children who did and did not achieve the

104 accelerometer wear time criteria $(p>0.05)$. On this basis we were satisfied that the data were

105 MAR rather than missing in a systematic manner. To replace the missing data, multiple

106 imputation ${ }^{18}$ consisting of 100 iterations was undertaken separately for boys' and girls'

107 missing weekday and weekend accelerometer data values using the Markov Chain Monte

108 Carlo algorithm. Following five imputations, pooled values were generated and subsequently

109 integrated into the data set prior to analysis.

110

111 The daily average $\min ^{\bullet} \mathrm{d}^{-1}$ spent in MVPA was calculated for each child. Sex-specific MVPA

112 quartile cut-off values (Table 1) were calculated to categorise boys and girls separately into 4

113 graded groups representing the least through to the most active children. The least active

114 children were grouped in quartile (Q) 1 with the rest grouped in Q2, Q3, or Q4 (i.e., Q4 as the

115 most active group). Descriptive statistics were calculated for all measured variables and

116 preliminary comparisons were made between PA groups using ANOVAs, MANOVAs, and

$117 \chi^{2}$ tests. The primary outcome variables were mean $\min ^{\bullet} \mathrm{d}^{-1}$ spent in ST, LPA, MPA, VPA,

118 and MVPA. These analyses and the multiple imputation were performed using IBM SPSS

119 Statistics version 20 (SPSS Inc., Chicago, IL) with alpha set at $p<0.05$.

120 For the main analyses multilevel linear regression analyses were computed separately for

121 boys and girls to analyze differences in outcome variables between weekdays and weekends.

122 Multilevel linear regression analyses accounted for the nested nature of the study design. A

123 three-level data structure was initially used where children were defined as the first level unit

124 of analysis, schools as the second level unit, and year of data collection as the third level unit

$125{ }^{21}$. Schools and years of data collection were included as levels in the analyses to control for

126 the effect that they could have on the children's activity ${ }^{21}$. 'Crude' exploratory models were 
127 initially constructed to determine whether schools and years of data collection were

128 significantly influential to warrant their inclusion as levels. If they were not, these levels were

129 removed resulting in models with either two or three level data structures. The necessity of

130 adding random slopes to these crude models was then evaluated by comparing differences

131 between the $-2 \log$ likelihood value with the value from the preceding model containing only

132 random intercepts, and computing a $\chi^{2}$ test with two degrees of freedom ${ }^{21}$. 'Adjusted'

133 analyses were completed using the final crude models but which also controlled for the

134 effects of BMI z-score and SES, which are known to potentially confound differences in

135 children's objectively measured PA ${ }^{22}$. Data were analyzed using MLwiN 2.20 software

136 (Centre for Multi-Level Modelling, University of Bristol, UK). Regression coefficients in the

137 models were assessed for significance using the Wald statistic with one degree of freedom ${ }^{21}$

138 and alpha was set at $p<0.05$.

139 Results.

140 Of the 818 children that returned written informed consent, three girls did not have BMI z-

141 scores due to missing anthropometric data, and three boys' and two girls' postcodes were

142 unavailable to calculate IMD scores. Thus, results were available from 810 children.

143 Participants were well matched in relation to age, stature, and IMD score regardless of PA

144 quartile (Table 1). Boys and girls in Q1 had significantly greater body mass than Q4 peers

145 ( $p<0.05)$, and a significantly higher percentage of Q1 boys were classed as overweight/obese,

146 than those in Q4 ( $p<0.05)$. Significantly more Q2-4 boys achieved recommended PA levels

147 than Q1 boys ( $p<0.001)$. Significantly fewer Q1-2 than Q3-4 girls met the PA guidelines

148 ( $p<0.01)$. Unadjusted weekday and weekend PA are presented in Table 2. As expected,

149 significant differences in ST, LPA, MPA, VPA, and MVPA were observed between Q1-4

150 boys, and these were generally most pronounced for weekdays ( $p<0.05$ to 0.001$)$. On 
151 weekdays and weekends significant between quartile differences in girls ST, LPA, MPA,

152 VPA, and MVPA were also apparent $(p<0.05$ to 0.001$)$.

153 The main multilevel analyses investigated time of week differences in PA (Table 3). Q2 boys spent longer in LPA on weekdays $(p<0.05)$. Q1 $(p<0.001)$ and Q2 boys $(p<0.01)$ did significantly more weekday MPA than at weekends. Boys in Q1-Q3 accumulated significantly more VPA and MVPA on weekdays than at weekends. In contrast, only small differences in weekday and weekend ST and PA at all intensities were observed for Q4 boys.

158 Similar patterns emerged from analyses of the girls' data with the exception of ST, which Q2 and Q3 girls engaged in more on weekdays than at weekends $(p<0.05)$. Q1 and Q2 girls spent significantly longer in weekday MPA than at weekends $(p<0.05)$. Girls in Q1-Q3 engaged in more VPA $(p<0.05)$ and MVPA $(p<0.01)$ on weekdays than weekends. The exception to this was Q4 girls who accumulated similar amounts of ST, and PA at all intensities between weekdays and weekends.

Discussion.

This study found that the most active boys, and in particular, girls, maintained their ST and PA levels across weekdays and weekends. In contrast Q1-Q3 children were more active and

167 less sedentary on weekdays than at weekends, with the exception of Q1-Q2 boys whose' ST

168 differed only by $\sim 3$ min. The decline in weekend PA observed in Q1-Q3 children concurs

169 with previous research demonstrating that early adolescents are less active and sedentary at weekends regardless of age and gender ${ }^{6}$. Moreover, for Q1-Q3 children the magnitude of differences in weekday-weekend ST, LPA, MPA, VPA, and MVPA was generally similar, suggesting that for most active children the weekend reductions in ST and PA were consistent regardless of their PA level ${ }^{9}$. Lower weekend ST and PA may be explained by various factors. Sleep duration can limit waking hours available for recreational pursuits, and at 
175 weekends this is longer than on weekdays ${ }^{23}$. Further, the familial influence on ST and PA 176 (e.g., presence of siblings, social support, modelling) may be more significant at weekends

177 than at other periods of the week when discretionary opportunities for PA exist (e.g., after 178 school) ${ }^{5,22}$. Parental concerns over child safety can also impact on weekend PA through 179 restrictions over children's local independent mobility ${ }^{24}$.

180 A novel aspect of our study was comparing weekday and weekend ST and PA of children 181 classified by PA level. No previous studies have investigated ST in this way, and of the few 182 PA studies that have, the commonly observed trend of lower weekend PA was apparent.

183 Nyberg and colleagues compared weekday and weekend Actiwatch count $\cdot \min ^{-1}$ between 6-10 184 year old Swedish children categorised into high, medium, and low activity tertiles ${ }^{9}$. Similar 185 declines in weekend PA for boys (14-19\% decrease) and girls (13-24\% decrease) were 186 reported regardless of activity tertile. In the ALSPAC study high and low active children 187 recorded marginally more ActiGraph count $\cdot \min ^{-1}$ on weekdays than at weekends ${ }^{10}$. Neither studies though reported PA defined by intensity. Using accelerometer count $\cdot \min ^{-1}$ as a PA outcome means that all recorded movement is defined as PA. This erroneously includes episodes of ST as well as LPA. LPA in youth is inversely associated with cardiometabolic

191 health markers ${ }^{25}$, although health gains from MVPA are greater ${ }^{25}$, hence why this threshold 192 underpins PA recommendations for public health. Intensity-specific PA was investigated by Jago et al. who observed a weekend reduction in

194 MVPA in the English children classed as 'high-active/low sedentary' ${ }^{11}$. This contrasts with 195 our finding that the most active boys and girls either maintained or did not significantly 196 reduce their weekday MVPA at the weekend. The exact reasons for this are unclear and 197 previous literature on this specific topic is scarce. Favourable biological correlates such as 198 body composition, motor skill capability, and fitness are established factors that predispose 199 children to engage in PA and sport ${ }^{26}$. Empirical evidence suggests that children and 
adolescents involved in organised sport engage in more MVPA during weekdays and weekends ${ }^{27}$, and those who participate in competitive sport are more likely to meet PA guidelines ${ }^{28}$. Based on the higher VPA and MVPA levels of Q4 children it is plausible that a significant proportion of their PA consisted of regular sport participation, some of which may have been competitive. During the school week multiple opportunities for structured sports and PA exist. When these opportunities are integrated into the school curriculum (e.g., physical education classes) and the extended school day (e.g., organised after-school activities on school premises) they are accessible to all children regardless of sports competence, thus optimising inclusivity and potential likelihood of participation ${ }^{29}$. Out of school weekday sporting opportunities however, are more likely be taken by children who

210 receive strong direct parental support (e.g., payment of club subscriptions, transport to and

211 from practice venues) and encouragement (e.g., positive verbal reinforcement and feedback)

$212^{22}$, and demonstrate greater sport competence ${ }^{30}$. Assuming Q4 children regularly engaged in

213 structured weekday sport that was partly facilitated by parental support and encouragement,

214 the known associations between sport participation and PA levels lead us to speculate that Q4

215 children may also have participated in weekend sports activities to a greater degree than their

216 less active peers ${ }^{28}$. The role of parents may be a key factor in explaining these findings, as a

217 recent study reported how children with greater parental support had less of a decline in

218 weekend MPA and VPA over a one year period ${ }^{7}$. Though the role of parents and family is

219 consistent across studies it explains only a modest proportion of variance in weekend PA ${ }^{8,22}$.

220 Thus, factors beyond the home and family are likely influences on children's weekend PA.

221 Future research should focus on establishing the multidimensional correlates of children's weekend PA to better understand the determining factors of this behaviour.

223 Strengths of this study included the use of imputation procedures to maximise the sample size. Furthermore, the high participation rate gave us confidence that the risk of participant 
recruitment bias was minimized. Children were blinded to their classification into PA quartiles which occurred retrospectively so as not to influence their activity behaviours. For the same reason, researchers were blinded to the PA groupings during data collection. ST and

PA were measured objectively and the analyses accounted for potential confounding variables and for the collection of data across different years in different schools. A study

230 limitation was the cross-sectional design which precluded us from knowing whether the maintenance of the Q4 children's weekend ST and PA was consistent across other times of the year and between years. Furthermore, the data were collected in autumn and winter when children's ST is higher and PA is lower than in spring and summer ${ }^{5}$. For this reason weekday-weekend patterns of ST and PA may differ when the climate is warmer and daylight hours longer. The data were representative of predominantly white British 10-11 year olds in one north-west England borough, and on this basis the findings cannot be generalised to other groups or areas. PA was measured using hip-mounted accelerometry which has inherent limitations related to accuracy of assessing PA on inclines, and during activities involving cycling, swimming, and upper body movements. Further, lack of consensus as to the most appropriate accelerometer PA cutpoints means that reported PA may have been overestimated. Resource constraints though meant it was not possible to calculate individually calibrated cutpoints which may provide more accurate PA data.

\section{Conclusion.}

244 ST and PA were highest on weekdays in all children except for the most active group who 245 maintained their ST and PA levels at weekends. These trends were particularly evident among Q4 girls. Whether the most active children would continue to maintain their weekdayweekend ST and PA patterns is unknown, and future research should investigate this through prospective study designs which encompass multidimensional correlates of weekend ST and 
and PA as well as specific periods of time when any declines in high active boys' and girls' weekend ST and PA occur.

252 Practical implications.

- Weekends are important to target for interventions because observed decreases in most children's PA are generally consistent regardless of age and gender.

- The least active children are most in need of weekend intervention strategies, through cost-effective and resource efficient approaches.

- Parental support is important to attenuate reductions in weekend PA and to reduce weekday ST.

Acknowledgements.

The funding support of Liverpool John Moores University and Wigan Council is acknowledged. The authors thank Helen Roberts and Alex Jones at Wigan Council, and the children and teachers who participated in this research.

\section{References.}

1. Chief Medical Officers. Start Active, Stay Active. A report on physical activity for health from the four home countries, London, Dept. Health, 2011.

2. Saunders TJ. The health impact of sedentary behaviour in children and youth. Appl Physiol Nutr Metab. 2014; 39:402. DOI: 10.1139/apnm-2013-0446.

3. Fairclough SJ, Beighle A, Erwin H, et al.. School day segmented physical activity patterns of high and low active children. BMC Public Health. 2012; 12(1):406.

4. Stanley R, Ridley K, Dollman J. Correlates of children's time-specific physical activity: A review of the literature. Int J Behav Nutr Phys Act. 2012; 9(1):50. 
272 5. King AC, Parkinson KN, Adamson AJ, et al. Correlates of objectively measured physical activity and sedentary behaviour in English children. Eur J Public Health. 2010. DOI: $10.1093 /$ eurpub/ckq104.

6. Steele RM, van Sluijs EM, Sharp SJ, et al. An investigation of patterns of children's sedentary and vigorous physical activity throughout the week. Int J Behav Nutr Phys Act. 2010; 7:88.

7. Corder K, Craggs C, Jones A, et al. Predictors of change differ for moderate and vigorous intensity physical activity and for weekdays and weekends: a longitudinal analysis. Int J Behav Nutr Phys Act. 2013; 10(1):69.

8. Fairclough SJ, Ridgers ND, Welk G. Correlates of children's moderate and vigorous physical activity during weekdays and weekends. J Phys Act Health. 2012; 9:129-137.

9. Nyberg GA, Nordenfelt AM, Ekelund U, et al. Physical activity patterns measured by accelerometry in 6- to 10-yr-old children. Med Sci Sports Exerc. 2009; 41:1842-1848.

10. Riddoch CJ, Mattocks C, Deere K, et al. Objective measurement of levels and patterns of physical activity. Arch Dis Child. 2007; 92:963-969.

11. Jago R, Fox KR, Page AS, et al. Physical activity and sedentary behaviour typologies of 10-11 year olds. Int J Behav Nutr Phys Act. 2010; 7:59.

12. Office for National Statistics Web site. Neighbourhood Statistics. Area: Wigan (Local Authority). Available at http://www.neighbourhood.statistics.gov.uk/dissemination/LeadTableView.do?a=7\&b $=276785 \& \mathrm{c}=$ Wigan $\& \mathrm{~d}=13 \& \mathrm{e}=16 \& \mathrm{~g}=356907 \& \mathrm{i}=1001 \times 1003 \times 1004 \& \mathrm{o}=1 \& \mathrm{~m}=0 \& \mathrm{r}=1$ $\underline{\& s}=1386240656770 \&$ enc $=1 \&$ dsFamilyId $=87 \& n s j \mathrm{~s}=$ true $\& n s c k=$ false \&nssvg $=$ false $\&$ nswid=1231. Accessed 1 October 2013. 1990. Arch Dis Child. 1995; 73:25-29. 
297 14. Cole TJ, Bellizzi MC, Flegal KM, et al. Establishing a standard definition for child overweight and obesity worldwide: international survey. BMJ. 2000; 320:1240-1244.

15. Department for Communities and Local Government. The English Indices of Deprivation 2007, Wetherby, Communities and Local Government Publications, 2008.

302 16. Evenson KR, Catellier DJ, Gill K, et al. Calibration of two objective measures of physical activity for children. $J$ Sports Sci. 2008; 26:1557-1565.

304 17. Trost SG, Loprinzi PD, Moore R, et al. Comparison of accelerometer cut-points for predicting activity intensity in youth. Med Sci Sports Exerc. 2011; 43:1360-1368.

18. Catellier DJ, Hannan PJ, Murray DM, et al. Imputation of missing data when measuring physical activity by accelerometry. Med Sci Sports Exerc. 2005; 37:S555S562.

19. Rich C, Geraci M, Griffiths L, et al. Quality control methods in accelerometer data processing: defining minimum wear time. PLoS One. 2013; 8:e67206.

20. Potthoff RF, Tudor GE, Pieper KS, et al. Can one assess whether missing data are missing at random in medical studies? Stat Methods Med Res. 2006; 15:213-234.

21. Twisk JWR. Applied Multilevel Analysis, Cambridge, Cambridge University Press; 2006.

22. McMinn AM, Griffin SJ, Jones AP, et al. Family and home influences on children's after-school and weekend physical activity. Eur J Pub Health. 2013; 23:805-810.

317 23. Thorleifsdottir B, Björnsson JK, Benediktsdottir B, et al. Sleep and sleep habits from childhood to young adulthood over a 10-year period. J Psychosom Res. 2002; 53:529537. 
24. Carver A, Timperio A, Hesketh K, et al. Are children and adolescents less active if parents restrict their physical activity and active transport due to perceived risk? Soc Sci Med. 2010; 70:1799-1805.

25. Carson V, Ridgers ND, Howard BJ, et al. Light-Intensity Physical Activity and Cardiometabolic Biomarkers in US Adolescents. PLoS One. 2013; 8:1-7.

26. Welk GJ. The Youth Physical Activity Promotion Model: A conceptual bridge between theory and practice. Quest. 1999; 51:5-23.

27. Machado-Rodrigues AM, Coelho e Silva MJ, Mota J, et al. Physical activity and energy expenditure in adolescent male sport participants and nonparticipants aged 13 to 16 years. J Phys Act Health. 2012; 9:626-633.

28. Silva G, Andersen LB, Aires L, et al. Associations between sports participation, levels of moderate to vigorous physical activity and cardiorespiratory fitness in childrenand adolescents. J Sports Sci. 2013; 31:1359-1367.

29. Stanley RS, Boshoff K, Dollman J. A qualitative exploration of the "critical window": factors affecting Australian children's after-school physical activity. J Phys Act Health. 2013; 10:33-41.

30. Ulrich BD. Perceptions of physical competence, motor competence, and particiaption in organized sport: their interrelationships in young children. Res Q Exerc Sport. 